\title{
A Manufacturable Achromatic Fresnel Lens For CPV
}

\author{
Stephen Askins, Guido Vallerotto, Marta Victoria, Rebeca Herrero, César Domínguez, Ignacio Antón, and \\ Gabriel Sala
}

\begin{abstract}
The CPV industry has moved to very high concentration ratios (1000X or more) but the commonly used Fresnel lenses are limited to concentration ratios closer to $200 \mathrm{X}$ by chromatic aberration, leading manufacturers to turn to secondary optical elements, which increase cost and complexity. Here we present the achromatic on glass (ADG) lens a novel achromatic lens design and manufacturing method that may be similar in cost to existing silicone on glass (SOG) lenses but with improved performance In addition, the paper also summarizes on the results of ray-trace simulations, and experimental results of the first prototype lenses, proving the viability of the design.

Index Terms - concentrator photovoltaics, primary optic, chromatic aberration, achromatic lens, achromatic doublet on glass.
\end{abstract}

\section{INTRODUCTION}

During the last decade, the explosive growth of multijunction solar cell efficiency has led to resurgence in concentrator photovoltaics (CPV). At the same time, a focus on keeping costs low has led most recent designs to use concentration ratios of $1000 \mathrm{X}$ or higher. These levels far exceed that which a Fresnel lens, the most common Primary Optical Element (POE) used in modern high-concentration photovoltaics, can achieve, due primarily to the effect of chromatic aberration. Commonly, a Secondary Optical Element (SOE) is used to further concentrate the light [1]. However, the use of a SOE adds cost, both for the optic self as well as for the additional assembly steps. Most importantly, it adds a critical optical interface that could be a factor in reducing reliability of the module as whole.

An alternative to a SOE may be to use an achromatic lens. Achromats are usually dismissed for CPV applications due to their complexity. Languy et. al. [2] proposed a doublet of a poly (methyl methacrylate) PMMA lens and a polycarbonate (PC) lens, however the manufacturability was not clear. In this work we propose the "Achromatic Doublet on Glass" (ADG) lens. This is an achromatic lens combining glass, plastic, and elastomeric materials and using standard industrial processes, which opens the door to economical achromatic Fresnel parquets for $\mathrm{CPV}$.

\section{BASIC OF ACHROMATS}

To produce an achromatic doublet, we must combine two lenses: a diverging, low dispersion lens, and a converging high dispersion lens. Dispersion (the variability of index of refraction with wavelength) can be typically summarized by the Abbe number. The Abbe number, $v$, compares the index of refraction at three wavelengths, $\lambda_{1}, \lambda_{2}$, and $\lambda_{3}$ :

$$
v=\frac{n\left(\lambda_{2}\right)-1}{n\left(\lambda_{3}\right)-n\left(\lambda_{1}\right)}
$$

For visible optics, the three wavelengths used are those that correspond to the Fraunhofer C, D, and F spectral line, however for a solar lens we should choose a spectral range corresponding to our cell's spectral response. As is well known, the achromatic condition is achieved when two lenses are combined such that the products of their Abbe and fnumber are equal but of opposite sign:

$$
f_{1} v_{1}=-f_{2} v_{2}
$$

And, assuming our achromat is a doublet, the equivalent focal distance of the lens can be found by:

$$
f_{e q}^{-1}=f_{1}^{-1}+f_{1}^{-2}
$$

Combining these two equations, we find the required fnumbers of the two lenses for a given ratio of Abbe numbers. As shown in Fig. 1, the smaller the ratio of $v_{2} / v_{1}$, the "slower" each individual lenses can be while preserving the achromatic condition. We must then find pairs of material with Abbe numbers as different as possible, because otherwise our lenses must both have very short focal lengths which will lead to optical losses.

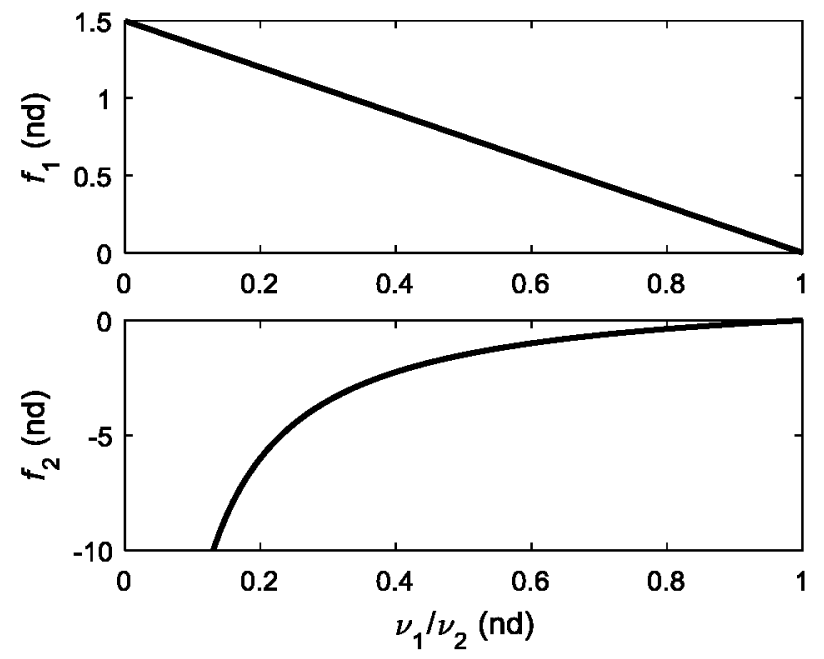

Fig. 1. Effect of Abbe number ratio $v_{2} / v_{1}$ on individual lens $\mathrm{f}-$ numbers, for $f_{e q}=1.5$. 


\section{ACHROMATIC DOUBLET ON GLASS}

Languy et. al. proposed using $\mathrm{PC}$ as a high dispersion material and PMMA as a low dispersion material [2], [3]. These are both well understood optical plastics that can be manufactured at high volume for low cost. However, if we attempt to combine them into a solar Fresnel lens we run into a difficulty. Of the three interfaces: (air to lens 1 , lens 1 to lens 2, and lens 2 to air) at least two must be structured as a Fresnel lens. Generally, in CPV, it is not desirable to place a Fresnel structure facing the sun, since this is the exterior of the module and a flat, cleanable surface is desired. However the only other option is to place a Fresnel structure at the interface between the two lenses, which requires manufacturing a matching pair of plastic lenses that can be fit together perfectly. Both the required molding precision and the assembly process may be prohibitive in cost.

In our proposed Achromatic Doublet on Glass (ADG) lens technology, a rigid lens made of high-dispersion thermoplastic, such as polycarbonate, is coupled with an elastomeric optical encapsulant, such as silicone. These materials have a sufficiently low dispersion to form an achromat with PC. We have identified a number of candidate materials that we are evaluating, the Abbe vs. index number of these materials is shown in Fig. 2.

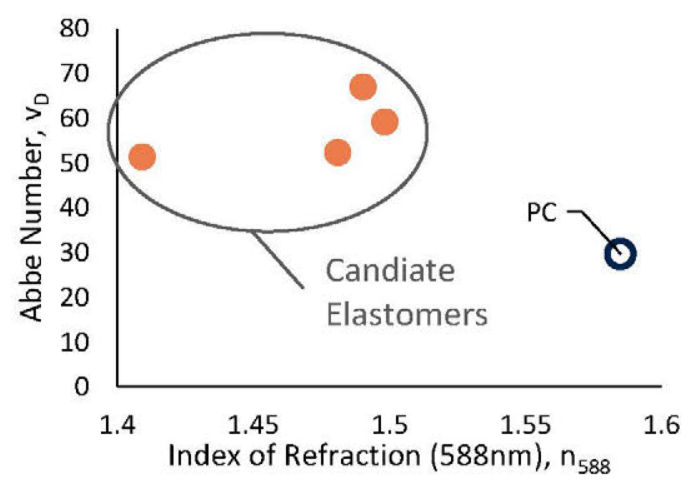

Fig. 2. Some of the candidate optical elastomers identified for use with the $\mathrm{ADG}$ concept.

The key advantage to using an encapsulant is that the two Fresnel interfaces can be injected molded onto either side of the plastic part, forming what we will refer to as a bi-Fresnel lens. This lens is then laminated to a flat glass plane using the encapsulant. As the encapsulant fills the space between the window and the plastic lens, it forms the shape of the desired low-dispersion lens in situ, and is then cured (Fig. 3). Therefore, we can place our second Fresnel interface between the two lenses without any difficulties in matching up rigid lenses, and preserve a flat surface towards the sun. ADG lenses additionally have the benefits of glass as an outward facing element. The rigidity, flatness, and scratch resistance of glass has been one of the primary reasons CPV manufacturers have shifted away from PMMA lenses to Silicone-on-Glass (SOG) the recent years.
The end-result is a manufacturing method for achromatic Fresnel lenses that is similar to a flat-plate solar panel lamination process. In comparison to the most common solar Fresnel manufacturing technique today (SOG), our achromatic lens will have the advantage that no costly tooling must be left in place during curing. And while the plastic lens must be manufactured in a previous step, either by plastic injection molding or hot embossing, we feel confident that the elimination of the long processing times at the lens assembly step should allow this method to be cost competitive with SOG, while offering better performance.

(a)

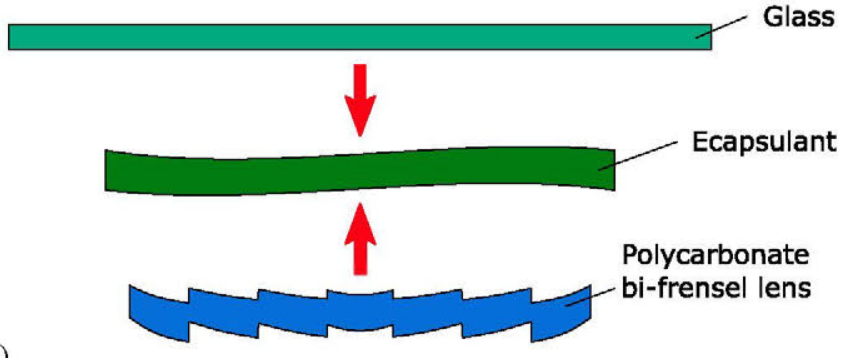

(b)

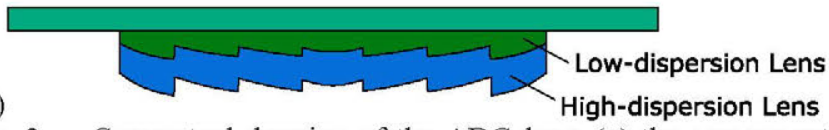

Fig. 3. Conceptual drawing of the ADG lens: (a) the components prior to the lamination process. (b) the laminated doublet.

\section{A. Design and predicted performance ADG Lenses}

The optical design is performed by imposing an "achromaticity condition" for two wavelengths: the Fresnel facets are designed in such a way that blue light and red light have exactly the same focal distance. The general design method for achromatic doublets is well documented [4], while a detailed disclosure relating to our specific design will be presented shortly [5].

The predicted focal distance versus wavelength of the lens, as calculated by an area-weighted application of geometrical optics for each facet, is shown in Fig. 4, as compared to a conventional SOG lens.

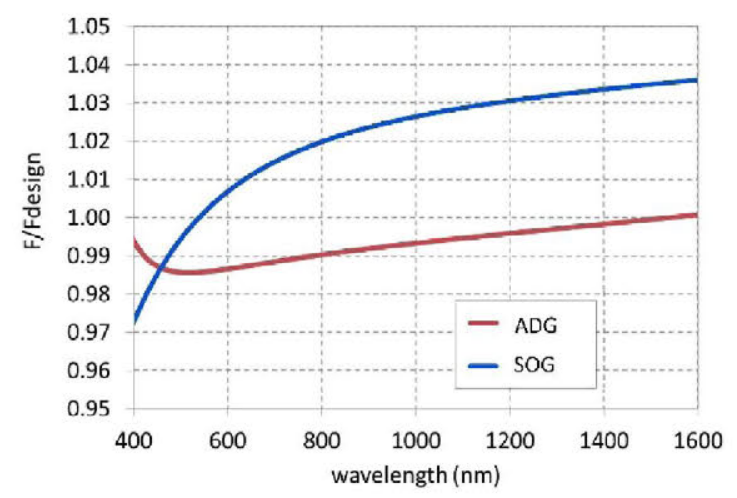

Fig. 4. Comparison of predicted focal distance with respect to wavelength. 


\section{RAY TRACING SimUlation}

A comprehensive set of ray-tracing simulations was performed in order to predict and analyze the performance of the ADG Fresnel lens previously described, as well as to compare its performance to an SOG lens of the same size and focal distance, and comparable Fresnel design parameters. This analysis will be described more fully in [5], while in this article we will only highlight the principle results.

\section{A.. Size of Generated Light Spot}

In this section we discuss the size of the generated light spot and the achromaticity behavior of ADG Fresnel lens. We simulated the top- and middle- subcells of a typical latticematched GaInP/GaInAs/Ge solar cell by implementing receivers with the appropriate spectral response. In this way it was possible to analyze ADG concentrating capability for blue light, converted by top subcells, and for red light, converted by middle subcells. The bottom subcell has not been considered because there is limited refractive index variation beyond $900 \mathrm{~nm}$, and as a consequence the lens behavior with light of more than $900 \mathrm{~nm}$ will be quite similar to its behavior between 650 and $900 \mathrm{~nm}$.

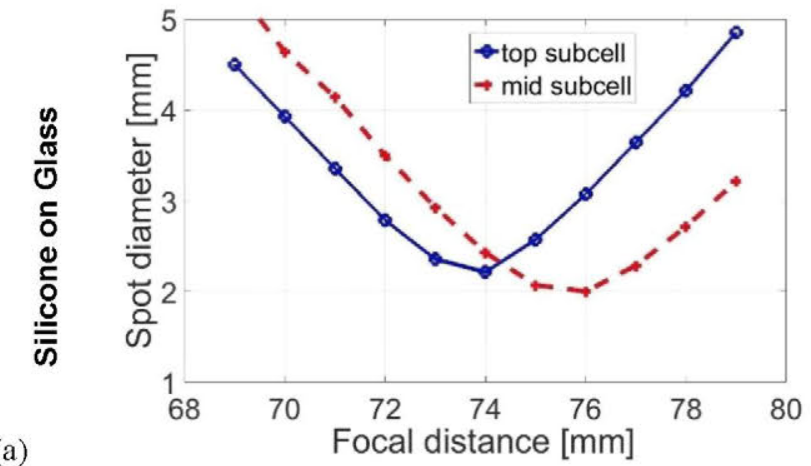

(a)
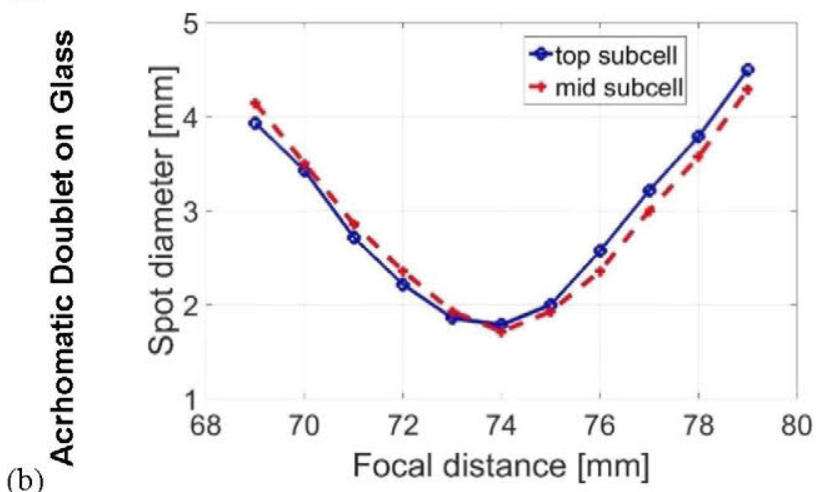

Fig. 5. Spot diameter predicted by ray-tracing as a function of the receiver displacement from the optimal focal distance (minimum spot). Spot diameter is defined as that including $99 \%$ of the energy. Red dashed lines represent spot diameters for longer wavelengths (those typically converted by middle subcell in MJ solar cells, i.e.:650-900nm) and blue continuous lines represent spot diameters for shorter wavelengths (those typically converted by top subcell, i.e.: $350-650 \mathrm{~nm}$ ).
For both lenses, the receiver (both top and middle) position was varied from five $\mathrm{mm}$ closer to the lens than the optimal position to five $\mathrm{mm}$ further from the optimal position, where optimal position is that which correspond the minimum spot size. The light "spot" diameter was defined as the radius was defined by $95 \%$ encircled energy, and are plotted for both simulated subcells in Fig. 5 ..

For the classic SOG Fresnel lens, the minimum of the two curves are displaced as shown in Fig. 5a. This is due to the chromatic aberration: since refractive index for short wavelength is higher, the focal point for blue light is closer to the lens than the one for the red light (remember Fig. 1) and so the minimum spot for blue light is displaced to the left (toward the lens) and the minimum spot for red light is displaced to the right (toward infinite). On the other hand, in Fig. 5b we see that, for the ADG Fresnel lens, the minimum spot for blue light corresponds exactly with the minimum spot for red light, demonstrating its achromaticity.

\section{B. Optical Efficiency versus Geometric Concentration}

We compared how the overall optical efficiency, defined as the percentage of the total luminous power transferred to a circular receiver placed at the optimum focal distance as determined from the previous studies, varied with receiver diameter. (Fig. 6). We note that for the lowest geometrical concentration ratios, the $\mathrm{ADG}$ produces slightly less optical efficiency, due to geometrical losses (ie. there two Fresnel surfaces instead of one, with attendant additional tip rounding and draft angle losses). However, if we examine the geometric concentration ratio that corresponds with the intersection of the two curves on Fig. 6, we see that SOG only outperforms $\mathrm{ADG}$ for concentration ratios less than approximately $250 \mathrm{X}$. Beyond this level, the SOG optical efficiency drops off sharply, while the ADG optical efficiency remains relatively constant to around $700 \mathrm{X}$ (because $\mathrm{ADG}$ as a relatively smaller spot size).

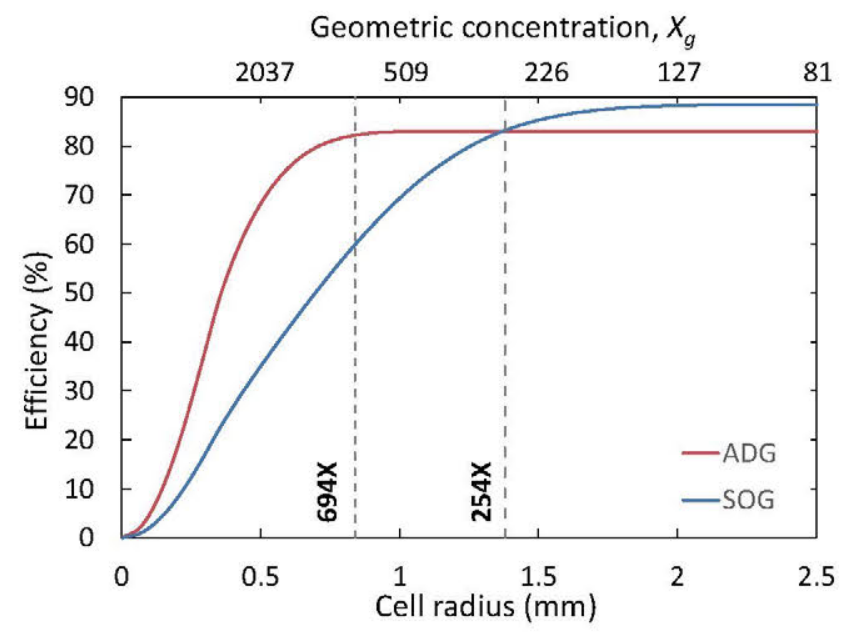

Fig. 6. Evolution of the optical efficiency for both SOG and ADG lens types versus the size of the simulated cell in a ray tracing simulation. The equivalent geometric concentration ratio is shown in the upper $\mathrm{x}$-axis. 


\section{PROTOYPE MANUFACTURE}

With the support of a European Horizon 2020 grant, CPV Match, we have designed and fabricated prototype achromatic Fresnel lenses for evaluation. The lens design chosen was $40 \times 40 \mathrm{~mm}^{2}$ with a 75 focal distance. This lens is the same size as an SOG lens that was provided by our project partners, Fraunhofer ISE. A rigid bifacial Fresnel lens was manufactured out of polycarbonate by Fresnel Optics GmbH according to our design. We used a custom $200 \mathrm{~mm} \times 200 \mathrm{~mm}$ laminator (Fig. 7) to encapsulate these lenses to small pieces of low-iron glass. As with any new processing technique, some fine-tuning was required, but within a few weeks of receiving the prototype plastic parts we had already produced the lens shown in Fig. 8 and we believe the ease with which we could proceed with encapsulation bodes well for the manufacturability prospects of this lens.

The lamination process is divided in three steps: degasification, lamination, and curing phase. An optimization of manufacturing parameters such as the duration of each step and temperature and pressure at which they are carried out has been realized. Using optimized parameters we could easily manufacture prototypes similar to those of Fig. 8, which has a very good transparency and it is air bubble free. Optimal parameters are related to the material we are currently using for the elastomeric layer.

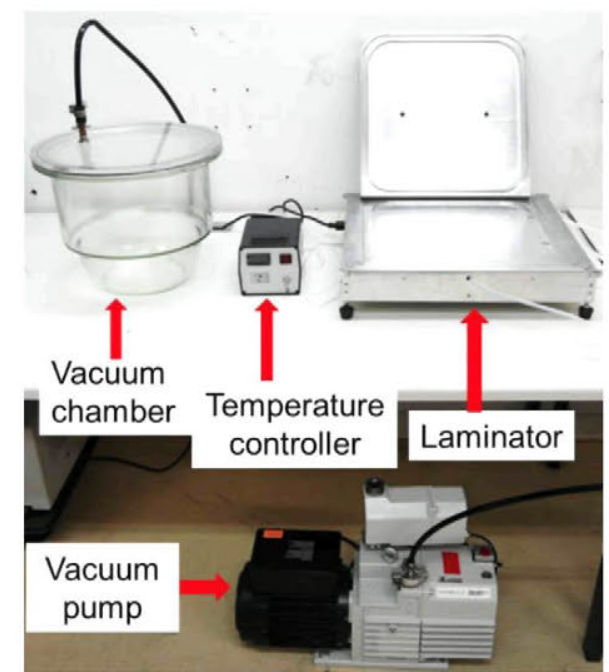

Fig. 7. Prototype laminator built at the IES and used to laminate the first ADG Fresnel lens prototypes, including that of Fig. 8.

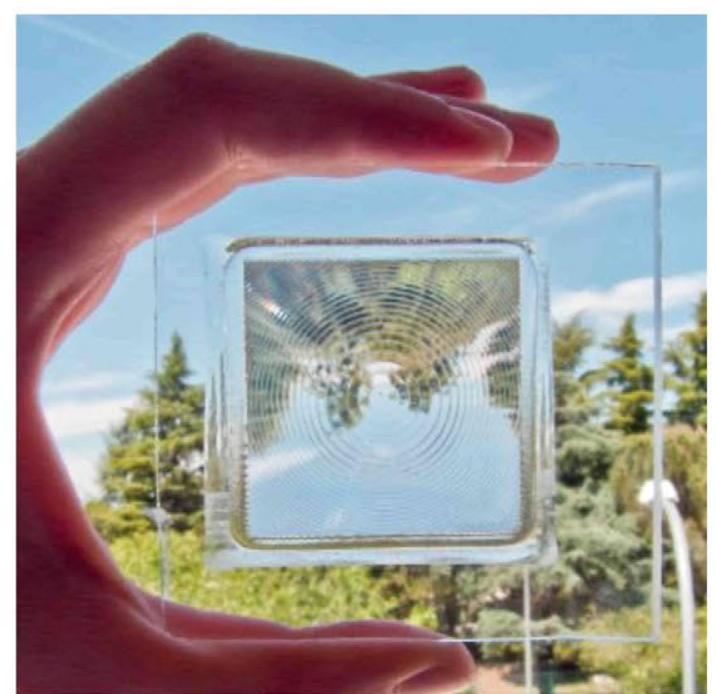

Fig. 8. An early ADG prototype laminated at the IES-UPM.

\section{EXPERIMENTAL}

At the IES, we have developed a suite of characterization methods for primary lenses [6] based on the use of our solar simulator for $\mathrm{CPV}$. We have used these methods to measure the spot size of the light in the two spectral ranges of interest: that corresponding to the top and middle subcells of a conventional GaInP/GaInAs/Ge lattice-matched multijunction cell, and compared the achromatic prototypes to a SOG prototype provided by Fraunhofer I.S.E

\section{A. Experimental Setup}

Fig. 9 shows a functional scheme of the experimental setup used to carry out experimental testing (Fig. 9a) and a photograph of the same system (Fig. 9b). The setup is composed of:

- A collimated light source having an angular aperture equal to $\pm 0.27^{\circ}$ composed of a lamp within an integrating sphere and a large area parabolic mirror used to collimate the light beam proceeding from the source.

- The sample lens (ADG or SOG) with a moving support through which it is possible to adjust the distance between lens and camera.

- Low pass or high pass filters with a cutoff of approximately $670 \mathrm{~nm}$.

- A charge coupled device (CCD) sensor. 
(a)

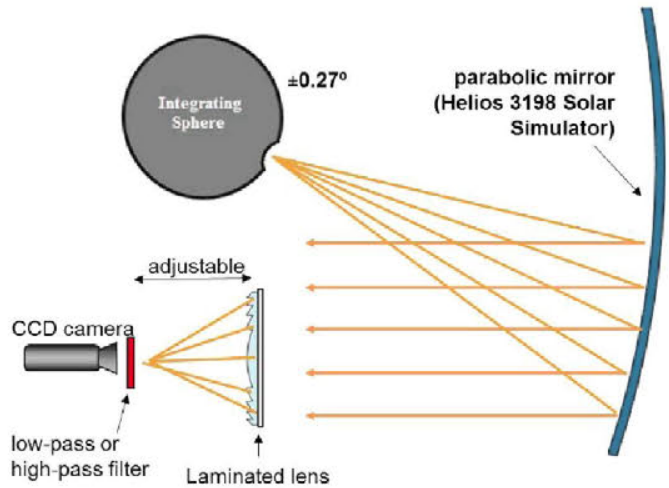

(b)

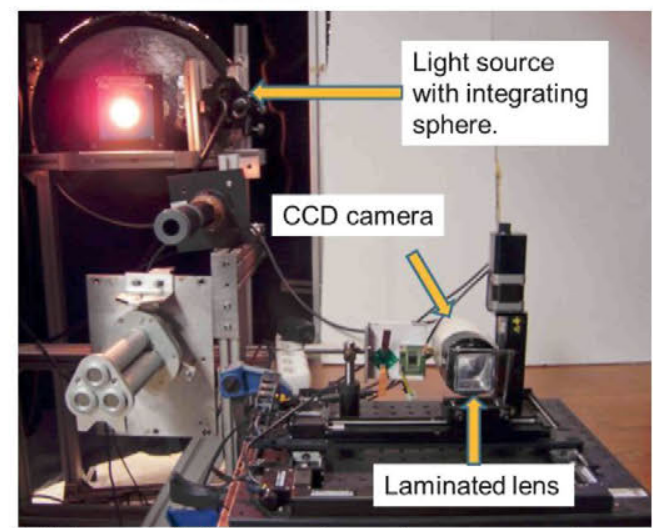

Fig. 9. a) Scheme of the experimental set-up used to carry out experimental testing. b) Photograph of the experimental setup and its components (light source with integrating sphere, lens sample, and CCD camera. Parabolic mirror and filters are not visible in this photograph).

The CCD sensor was placed near the focus of the sample lens, and by processing the resulting image it is possible to get the diameter of the generated light spot. Low- and high-pass diffractive filters with appropriate cut-off frequencies were used to selectively image only light converted by top or middle subcells depending on which filter has been used. By moving the lens support with respect to the CCD array, it is possible to move the lens closer or further from the camera. In this way we were able to locate the optimal position (minimum spot diameter) for both tested lenses and for both subcells.

\section{B. Experimental Results}

The evolution of the spot diameter as a function of the lens position with respect to the camera for both lenses and subcells is shown in Fig. 10. This figure is similar to Fig 3 but represents empirical data instead of ray tracing simulation results.

First, by comparing Fig. 9 with Fig. 3, we can see that the achromatic behavior of the ADG lens predicted by ray tracing simulations has been verified by experimental results. In fact, in Fig. 9 it is possible to appreciate the same displacement between minimum points of blue and red curves for the SOG lens while, as expected, minimum for blue and red curves for the ADG lens coincides. This confirms correctness and reliability of our design method and manufacturing process.

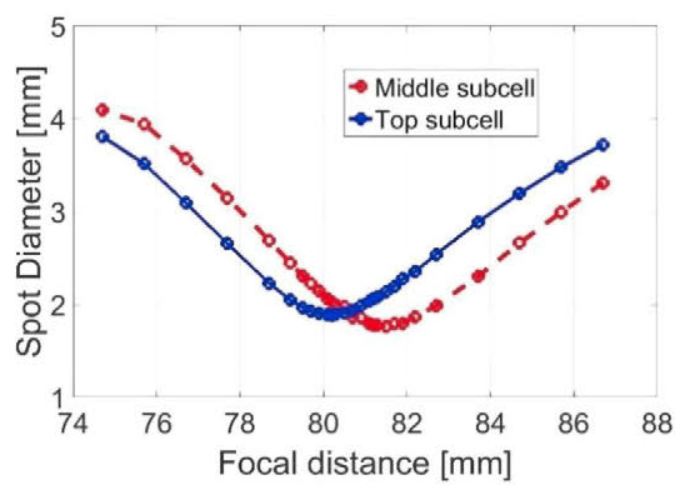

(a)

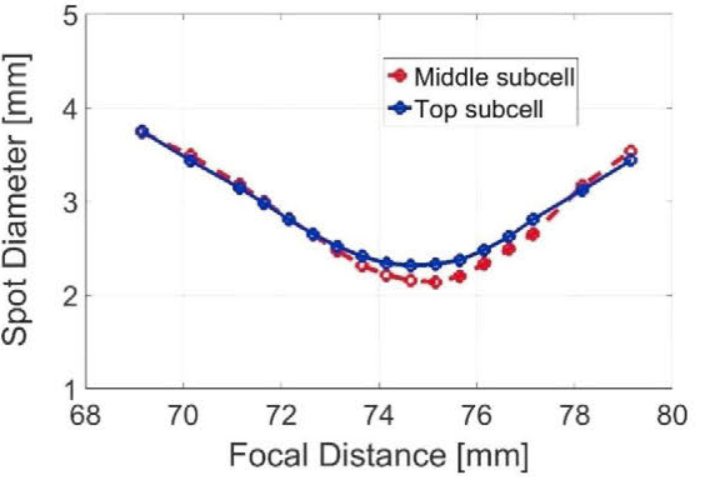

(b)

Fig. 10. Measured spot diameter as a function of the receiver displacement from the optimal focal distance (minimum spot). Spot diameter is defined as that including $95 \%$ of the energy. Red dashed lines represent spot diameters for longer wavelengths (those typically converted by middle subcell in MJ solar cells, i.e.:650-900nm) and blue continuous lines represent spot diameters for shorter wavelengths (those typically converted by top subcell, i.e.: $350-650$ $\mathrm{nm})$.

We observe that while ray tracing predicts that the minimum spot size produced by ADG lenses will be smaller than those of SOG, the experimental results presented above show the opposite. This is due to two factors:

1. The SOG Fresnel lens used in ray-tracing simulation has the same geometric parameters than the ADG Fresnel lens (f-number in the diagonal dimension equal to 1.33) while the SOG Fresnel lens provided by Fraunhofer I.S.E. has a higher focal distance (fnumber in the diagonal dimension equal to 1.45). It has been shown that the relation between maximum attainable concentration and $\mathrm{f}$-number has a maximum for an f-number value of about two [7] and decrease sharply for lower values.

2. Second, the reference SOG designed for ray-tracing simulation, as well as the present ADG lens design, have flat facets in order to ease design and potential manufacturing process. The SOG Fresnel lens provided by Fraunhofer I.S.E. features curved facets resulting in an improved concentrating power. By incorporating curved facets into our design we should be able to reduce our spot size further. 


\section{CONCLUSIONS}

We have presented a novel design for an achromatic Fresnel lens applicable to CPV, and verified its validity both via simulation and experimental measurements. The ease with which we were able to prototype this lens bodes well for its future manufacturability.

A novel achromatic lens concept applicable to HCPV and its manufacturing method have been presented. The proposed manufacturing process is at the same time simple, costeffective, and reliable. The primary manufacturing steps are the plastic injection of a bi-Fresnel lens and its lamination with an optical elastomer to a glass substrate. The ease with which we were able to prototype the lens suggests that the method will be easily scalable to volume production and its overall cost will be comparable with the cost of the manufacturing process currently used to fabricate classic SOG Fresnel lenses.

We have validated the achromaticity of this lens design with both ray-tracing and experimental results. After these initial results, future steps for ADG development include the improvement of the design (i.e. including curved facets) and of the manufacturing method in order to increase both optical efficiency and maximum attainable concentration. Additionally we are already beginning reliability testing on the lens design.

\section{ACKNOWLEDGEMENT}

This work has been supported by both Spanish Ministry of Economy and Competitiveness under the "Acromalens" project (ENE2013-45229-P) and has received funding from the European Union's Horizon 2020 research and innovation program within the project CPVMatch under grant agreement No 640873 .

\section{REFERENCES}

[1] M. Victoria, C. Domínguez, I. Antón, and G. Sala, "Comparative analysis of different secondary optical elements for aspheric primary lenses," Opt. Express, vol. 17, no. 8, pp. 6487-6492, Apr. 2009.

[2] F. Languy and S. Habraken, "Performance comparison of four kinds of flat nonimaging Fresnel lenses made of polycarbonates and polymethyl methacrylate for concentrated photovoltaics," Opt. Lett., vol. 36, no. 14, p. 2743, Jul. 2011.

[3] F. Languy, K. Fleury, C. Lenaerts, J. Loicq, D. Regaert, T Thibert, and S. Habraken, "Flat Fresnel doublets made of PMMA and PC: combining low cost production and very high concentration ratio for CPV," Opt. Express, vol. 19, no. S3, p. A280, May 2011.

[4] E. Hecht, Optics, Third Edition. Addison Wesley Longman, Inc., 1998.

[5] G. Vallerotto, M. Victoria, S. Askins, R. Herrero, C. Domínguez, I. Antón, and G. Sala, "Design and modelling of a cost-effective achromatic Fresnel lens for concentrating photovoltaics," Opt. Express, To be submitted.

[6] M. Victoria, S. Askins, R. Herrero, I. Antón, and G. Sala, "Assessment of the optical efficiency of a primary lens to be used in a CPV system," Sol. Energy, vol. 134, pp. 406-415, Sep. 2016.

[7] M. Victoria, "New concepts and techniques for the development of high-efficiency concentrating photovoltaic modules," $\mathrm{PhD}$, E.T.S.I. Telecomunicación (UPM), 2014. 